\title{
ESTUDO EPIDEMIOLÓGICO DA LEISHMANIOSE VISCERAL NA ÁREA URBANA E RURAL DO MUNICÍPIO DE TEÓFILO OTONI - MINAS GERAIS
}

\section{EPIDEMIOLOGICAL STUDY OF VISCERAL LEISHMANIOSIS IN THE URBAN AND RURAL AREA OF THE MUNICIPALITY OF TEÓFILO OTONI- MINAS GERAIS STATE}

\author{
Danila Gusmão Azevedo \\ Graduanda em farmácia, UNIPAC-TO - Brasil. \\ danila_gusmao@hotmail.com.br \\ Miriã de Oliveira Santos \\ Graduanda em farmácia, UNIPAC-TO - Brasil. \\ mirisantosrs@hotmail.com.br \\ Andreia Teixeira Oliveira Santos \\ Farmacêutica- Doutora em Biocombustíveis. UNIPAC-TO. \\ andreia.compbyte@gmail.com. \\ Lucio Onofri \\ Mestre em Zootecnia -Professor da Unipac TO \\ lucioonofri@gmail.com
}

\section{RESUMO}

As Leishmanioses são um complexo de doenças que acometem o homem e diferentes espécies de mamíferos silvestres e domésticos das regiões tropicais e subtropicais, causadas por protozoários digenéticos (heteroxenos) do gênero Leishmania, pertencentes à ordem Kinetoplastida, da família Trypanosomatidae, transmitidos através da picada de diferentes espécies de flebotomíneos infectados. A doença é conhecida mundialmente por ser uma zoonose de grande incidência, de ampla distribuição e com alto índice de mortalidade, presente em 12 países. Atualmente, a leishmaniose encontrase em expansão, com aumento de novos casos notificados entre os períodos de 20012017, totalizando 59.769 casos novos, resultando em uma média de 3.516 casos por ano, sendo $96 \%$ (57.582) deste quantitativo reportado no Brasil. O objetivo desta pesquisa foi analisar através de um estudo retrospectivo, com abordagem descritiva $\mathrm{e}$ quantitativa, a situação da Leishmaniose Visceral no município de Teófilo Otoni Minas Gerais, Brasil, no período de 2014 a 2018. Para tal, foram analisados os casos notificados no banco de dados do Departamento de Informática do Sistema Único de 
Saúde (DATASUS). A análise da doença na região demonstrou uma expansão geográfica da infecção e um aumento expressivo dos casos humanos (100\%) nos anos de 2016 a 2018, seguindo o padrão de expansão nacional.

Palavras-chave: Leishmaniose visceral; Epidemiologia; Doenças negligenciadas.

\begin{abstract}
Leishmaniasis are a complex of diseases that affect man and diferente species of wild manamals if tropical and subtropical regions, caused by digenetic protozoa (heteroxenes), genus leishmania, blongs to Kinetoplastida order of trypanosomatidae family. The ilness is worldwide knouen for being a zoonosis of great incidence widely distribuited and with high mortality rate, presente in twelve countries. Currently, leishmaniasis is expanding, with increase in new cases reported between the periods of 2001 - 2017, totalizing 59. 769 new cases per year, being 96\% (57.582) of this quantitave reported in Brazil. The aim of this research was to analyse through a retrospective study, with a descriptive and quantitative approach, the situation of Visceral Leishmaniasis in Teofilo Otoni city - Minas Gerais, Brazil, in the period from 2014 to 2018. For this, were analyzed cases notified in database of Computer departament of Sistema único de Saude (DATASUS). The analysis of disease in region shwed a geografical expansion of this infection and an expressive increase in human cases (100\%) from 2016 to 2018, follow ing the nacional expansion pattern.
\end{abstract}

Keywords: visceral leishmaniasis, epidemiology, neglected diseasis.

\title{
1 INTRODUÇÃO
}

As Leishmanioses são um complexo de doenças que acometem o homem e diferentes espécies de mamíferos silvestres e domésticos das regiões tropicais e subtropicais (RONDON, 2007; WORLD HEALTH ORGANIZATION, 2012; NUNES et al., 2010). São causadas por protozoários digenéticos (heteroxenos) do gênero Leishmania, pertencentes à ordem Kinetoplastida, família Trypanosomatidae, transmitidos através da picada de diferentes espécies de flebotomíneos infectados (BASANO; CAMARGO, 2004; MARZOCHI, 1992; SHAW et al., 1987; YOUNG; DUNCAN, 1994). De acordo com a espécie do flebotomíneo, podem produzir manifestações clínicas distintas, caracterizadas por Leishmaniose Tegumentar (LT) ou Leishmaniose Visceral (LV).

A LT produz manifestações cutâneas, mucocutâneas ou cutâneas difusas, 
causadas pelos protozoários Leishmania mexicana, L. brasiliensis e L. tropica. A LV caracteriza-se por manifestações variáveis que incluem febre alta, tosse improdutiva, diarreia e comprometimento de órgãos internos, especialmente o fígado e o baço, causadas pelos protozoários L. chagasi (MARZOCHI, 1992).

Nas Américas, as leishmanioses estão presentes em 12 países. A forma clínica mais severa e quase sempre fatal, se não tratada é a LV, com evolução abrupta, levando ao enfraquecimento geral do paciente e aumentando a suscetibilidade a infecções secundárias e hemorragias (CORRÊA; CORRÊA, 1992; VERONESI; FOCACCIA, 1996). No Brasil, a LV acomete pessoas de todas as idades, com incidência de $80 \%$ dos casos em crianças menores de 10 anos de idade nas áreas endêmicas (BRANDÃO FILHO et al., 2002).

A Leishmaniose é uma doença emergente, conhecida mundialmente por ser uma zoonose de grande incidência, de ampla distribuição e com alto índice de mortalidade. Segundo o programa de controle de doenças da Organização Mundial da Saúde (OMS, 2019), a doença atualmente está entre as endemias tropicais prioritárias, apresentando grande expansão pelo país em todas as regiões. No ano de 2017 foram registrados 4.239 novos casos de LV, representando um aumento regional de $26,4 \%$ quando comparado ao ano 2016, dado ao aumento de $28 \%$ dos casos no Brasil (OMS, 2019).

Os casos de Leishmaniose são registrados em todos os estados no Departamento de Informática do Sistema Único de Saúde (DATASUS), por intermédio do SINAN (Sistema de Informação de Agravos de Notificação), buscando-se a identificação da expansão dos casos por região. A vigilância epidemiológica é responsável pela análise de qualquer caso notificado suspeito ou confirmado no SINAN. A partir das informações coletadas de cada região, busca-se estratégias de ação que visem impedir a disseminação da doença.

A estratificação de riscos é uma importante ferramenta para a vigilância, visto que proporciona um melhor conhecimento sobre o problema de saúde, o qual oferece 
subsídios aos gestores e profissionais da saúde para a adoção de ações e direcionamento de áreas prioritárias a serem trabalhadas. Neste contexto, diante do quadro crescente de notificações de casos de LV humana em todas as regiões do Brasil, foi realizado um estudo com o objetivo de descrever a situação epidemiológica atual da Leishmaniose Visceral Humana no município de Teófilo Otoni, Minas Gerais, de forma a subsidiar o planejamento de futuras estratégias de prevenção e, ou, controle dessas enfermidades pela Secretaria Municipal de Saúde da região.

O cenário de estudo traz características geográficas, climáticas e socioeconômicas significativas para o desenvolvimento do ciclo de transmissão do agente etiológico da LV, sendo uma região classificada como endêmica para Leishmaniose.

\section{METODOLOGIA}

O presente trabalho é uma pesquisa retrospectiva, com abordagem descritiva e quantitativa, realizada por meio do levantamento situacional da Leishmaniose como problema de saúde pública, no município de Teófilo Otoni - MG., no período de 2014 a 2018.

O município de Teófilo Otoni pertencente à mesorregião do Vale do Mucuri, em Minas Gerais. O Vale do Mucuri está localizado na região nordeste do estado de Minas Gerais, com limites com os Estados da Bahia e do Espirito Santo. Atualmente o município é bastante urbanizado, com regiões comerciais e residenciais. Todavia, segundo Martins (2010), a cidade está localizada onde originalmente havia uma densa Mata Atlântica, com árvores de até 30, 40 metros de altura.

Segundo dados do censo realizado no ano de 2010, o município possui uma população de 134.745 habitantes e unidade territorial de 3.242.270 Km² (IBGE, 2014). 
O clima na bacia do Rio Mucuri é do tipo tropical úmido, com verão intenso (MARTINS, 2010).

A pesquisa foi realizada por intermédio de uma busca sistemática de artigos acadêmicos, contemplando o objeto do estudo proposto e da relevância para a análise proposta. Foram também, consultados os dados disponíveis em sítios, como o banco de dados do Departamento de Informática do Sistema Único de Saúde (DATASUS), ao qual estão anexadas as informações do SINAN (Sistema de Informação de Agravos de Notificação). Utilizou-se o programa DATASUS com diferentes filtros ao banco de dados para colher informações referentes à frequência anual da LV no município de Teófilo Otoni; e o perfil das pessoas infectadas (sexo, faixa etária, zona de residência).

Os cálculos de indicadores epidemiológicos da LV, do quinquênio em estudo, foram realizados com intuito de verificar o coeficiente de incidência $\left(n^{\mathbf{o}}\right.$ de casos novos da doença x 10n/população local do mesmo período). Após a coleta dos dados, foram elaborados gráficos por intermédio do programa Excel 14.0.

\section{RESULTADOS E DISCUSSÃO}

Segundo dados da OMS (2017), foi observado uma expansão geográfica da doença em países sul americanos como Argentina, Colômbia, Paraguai, Venezuela e Brasil (Figura 1). O total de novos casos da LV no Brasil no ano de 2017 foi de 4.239 , representando um aumento regional de $26,4 \%$ quando comparado ao ano de 2016, dado ao aumento de $28 \%$ dos casos nacionais. A incidência de LV nas Américas foi de 5,23 e 0,74 casos por 100.000 habitantes, considerando respectivamente, a população de áreas de transmissão e a população total dos países com ocorrência de casos de LV. 
Figura 1: Número, Proporção de casos e Incidência de leishmaniose visceral segundo países, Américas nos anos de 2015-2017.

\begin{tabular}{|c|c|c|c|c|c|c|c|c|c|c|c|c|}
\hline Paises & $\mathrm{N}^{*}$ & $\%$ & $\begin{array}{l}2015 \\
\text { Incid. Pop } \\
\text { Risco }\end{array}$ & $\begin{array}{l}\text { Incid. } \\
\text { Geral }^{2}\end{array}$ & $\mathrm{~N}^{*}$ & $\%$ & $\begin{array}{l}2016 \\
\text { Incid. Pop } \\
\text { Risco }^{1}\end{array}$ & $\begin{array}{l}\text { Incid. } \\
\text { Geral }^{2}\end{array}$ & $\mathrm{~N}^{*}$ & $\%$ & $\begin{array}{l}2017 \\
\text { Incid. Pop } \\
\text { Risco }^{2}\end{array}$ & Incid. Geral $^{2}$ \\
\hline Brasil & 3.289 & 95.2 & 4.09 & 2.54 & 3200 & 95.41 & 4.88 & 1.55 & 4114 & 97.05 & 5.53 & 1.98 \\
\hline Paraguai & 92 & 2.7 & 3.01 & 2.36 & 64 & 1.91 & 2.42 & 1.02 & 34 & 0.80 & 2.1 & 0.53 \\
\hline Venezuela & 37 & 1.1 & 1.24 & 0.34 & 33 & 0.98 & 1.03 & 0.11 & 40 & 0.94 & 1.33 & 0.13 \\
\hline Colômbia & 21 & 0.6 & 7.04 & 0.63 & 37 & 1.10 & 3.54 & 0.34 & 29 & 0.68 & 3.44 & 0.06 \\
\hline Argentina & 8 & 0.2 & 0.89 & 0.67 & 11 & 0.33 & 0.72 & 0.03 & 9 & 0.21 & 1.07 & 0.02 \\
\hline Honduras & 6 & 0.2 & 2.4 & 0.34 & 7 & 0.21 & 2.25 & 0.08 & 8 & 0.19 & 2.48 & 0.09 \\
\hline El Salvador & 0 & 0.0 & 0.0 & 0.0 & 0 & 0.00 & 0.0 & 0.00 & 2 & 0.05 & 4.4 & 0.03 \\
\hline Guatemala & 2 & 0.1 & 1.9 & 1.9 & 2 & 0.06 & 2.2 & 0.01 & 2 & 0.05 & 5.4 & 0.01 \\
\hline México & 1 & 0.0 & 4.3 & 0.1 & 0 & 0.00 & 0.0 & 0.00 & 1 & 0.02 & 5.4 & 0.00 \\
\hline Total & 3.456 & 100.0 & 5.07 & 2.42 & 3354 & 100.00 & 4.51 & 1.04 & 4239 & 100.00 & 5.23 & 0.74 \\
\hline
\end{tabular}

Fonte: SisLeish-OPAS/OMS: Dados reportados pelos Programas Nacionais de Leishmanioses /

Serviços de vigilância.

${ }^{1}$ Incidência por 100.000 hab. considerando a população das zonas de transmissão de LV nos países e regiões.

${ }^{2}$ Incidência por 100.000 hab. considerando a população total dos países com transmissão de LV

A incidência da LV observada no cenário do presente estudo durante o período de 2014 a 2018 foi de 56 casos notificados (Tabela 1), com aumento significativo de casos nos três últimos anos analisados (2016, 2017 e 2018).

TABELA 1: Frequência de casos de Leishmaniose Visceral no município de Teófilo Otoni nos anos de 2014 a 2018

\begin{tabular}{cc}
\hline Ano & Número de casos notificados \\
\hline 2014 & 7 \\
2015 & 6 \\
2016 & 18
\end{tabular}


FONTE: Dados da própria pesquisa

Do quantitativo de casos identificados através das fichas de notificação, foram separados os casos relatados na zona urbana da cidade e da zona rural. Na Tabela 2 são organizados os casos notificados em cada bairro do município, enquanto que na Tabela 3, observa-se o quantitativo de casos notificados na zona rural. Assim, em análise à Tabela 2, observa-se uma maior incidência nos bairros Bela Vista (19,5\%), São Cristóvão (10,8\%), seguidos pelos bairros Palmeira e Concórdia (ambos 6,5 \%); Cidade Alta, Fátima, Viriato, Frei Dimas, Taquara, Centro (com 4,3\%); Jardim Iracema, São Jacinto, Marajoara, Indaiá, Eldorado, Manoel Pimenta, Matinha, Alto Cemitério, Serra Verde, Felicidade, Novo Horizonte, Tancredo Neves (2,1\%). Dois bairros não foram identificados na ficha de notificação SINAM, totalizando (4,3\%). Observa-se uma incidência de $82,1 \%$ dos casos notificados localizados nas áreas urbanas do município de Teófilo Otoni. Na zona rural (Tabela 3), o quantitativo foi de 10,7\% dos casos notificados na região do estudo.

TABELA 2: Frequência de casos de Leishmaniose Visceral no município de Teófilo, classificados por bairros do município

\begin{tabular}{cccccccc}
\hline Bairros notificados & \multicolumn{8}{c}{$\begin{array}{c}\text { Número de casos por ano de } \\
\text { notificação }\end{array}$} & $\begin{array}{c}\text { Total de notificações } \\
\text { por bairro e período }\end{array}$ \\
\hline & $\mathbf{2 0 1 4}$ & $\mathbf{2 0 1 5}$ & $\mathbf{2 0 1 6}$ & $\mathbf{2 0 1 7}$ & $\mathbf{2 0 1 8}$ & \\
\hline Palmeiras & 01 & x & x & 01 & 01 & 03 \\
Cidade Alta & 02 & x & x & x & x & 02 \\
São Cristóvão & 01 & 01 & 03 & x & x & 05 \\
Jardim Iracema & 01 & x & x & x & x & 01
\end{tabular}


Fátima

Viriato

São Jacinto

Marajoara

Bela Vista

Indaiá

Frei Dimas

Taquara

Concórdia

Eldorado

Centro

Manoel Pimenta

Matinha

Alto Cemitério

Serra Verde

Felicidade

Novo Horizonte

Tancredo Neves nas fichas $\begin{array}{lllllll}\text { Bairros não identificados } & \mathrm{X} & \mathrm{x} & 02 & \mathrm{x} & \mathrm{x}\end{array}$

$$
01 \quad \mathrm{x} \quad 01 \quad \mathrm{x} \quad \mathrm{x}
$$

$$
\begin{array}{lllll}
\mathrm{X} & 01 & \mathrm{X} & 01 \quad \mathrm{X}
\end{array}
$$

$\begin{array}{llllll}X & 01 & \mathrm{X} & \mathrm{X} & \mathrm{X}\end{array}$

$\begin{array}{lllll}X & 01 & 04 & 02 & 02\end{array}$

01

$\begin{array}{llllll}\text { X } & \text { X } & 01 & \text { X } & \text { X }\end{array}$

$\begin{array}{lllll}\mathrm{X} & \mathrm{X} & 01 & \mathrm{X} & 01\end{array}$

$\begin{array}{llllll}\mathrm{X} & \mathrm{X} & 01 & \mathrm{X} & 01\end{array}$

03

$\begin{array}{lllll}\mathrm{X} & \mathrm{X} & 02 & \mathrm{X} & 01\end{array}$

$\begin{array}{llllll}01 & \mathrm{x} & \mathrm{x} & \mathrm{x} & \mathrm{x}\end{array}$

$\begin{array}{lllll}X & X & X & 01 & 01\end{array}$

01

01

$\begin{array}{lllll}\mathrm{X} & \mathrm{X} & \mathrm{X} & 01 \quad \mathrm{X}\end{array}$

01

$\begin{array}{lllll}X & \mathrm{X} & \mathrm{X} & 01 & \mathrm{X}\end{array}$

01

$\begin{array}{llllll}X & X & X & X & 01\end{array}$

01

$\begin{array}{llllll}X & X & X & X & 01\end{array}$

01

$\begin{array}{llllll}X & X & X & X & 01\end{array}$

$\begin{array}{llllll}X & X & X & X & 01\end{array}$

01 02 
FONTE: Dados da própria pesquisa

O maior percentual de casos de LV por zona de residência foi encontrado na área urbana, com um total de $82,1 \%$ de notificações.

Segundo Basano e Camargo (2004), os principais fatores responsáveis pelo crescente número de casos na zona urbana são os processos migratórios, precariedades em saneamento básico, baixa condição socioeconômica, desmatamento desenfreado para construção de assentamentos, estradas e fábricas e crescimento da agropecuária, entre outros, destruindo e invadindo o habitat do flebotomíneo vetor da Leishmaniose. Nesse último fator, a população cria uma proximidade com o habitat do vetor, sejam nas áreas urbanas pelo crescimento desordenado e consequente redução de vegetação, como também nas áreas rurais, locais normalmente utilizados pela população para uso recreativo.

De acordo com Martins (2010), a cidade está localizada onde originalmente havia uma densa Mata Atlântica, com árvores de até 30, 40 metros de altura. Tal fator pode estar associado às características endêmicas da região.

Segundo Costa (2011), o processo desordenado de ocupação urbana resultou em condições precárias de vida e destruição ambiental, fatores que também podem ter influenciado a emergência da doença no meio urbano. Neste contexto, para a efetividade das ações de prevenção e controle tanto da LV, torna-se imprescindível a intervenção em localidades onde a doença é endêmica, privilegiando o município, com ênfase na articulação e integração das ações na organização dos sistemas de saúde municipais (WHO 2011; CHAPPUIS et al., 2007), que tradicionalmente ocorrem de forma fragmentada (HARTZ E CONTANDRIOPOULOS, 2004; MENDES, 2010).

Outro fato a ser considerado no aumento expressivo dos casos de LV nos últimos anos é a prévia infecção canina associada à ocorrência de casos humanos (COSTA, 2008). O cão doméstico tem sido apontado em diversos estudos como o 
principal reservatório domiciliares e peridomiciliares de L. chagasi, sendo responsabilizado pelo surgimento e manutenção de focos endêmicos e epidêmicos da doença nos grandes centros urbanos (SILVA et al., 2001; SILVA et al., 2007). A presença de vetor e a manutenção do animal infectado próximo a estas áreas endêmicas contribuem para a dispersão da doença constituindo um risco para a população.

O tratamento de cães não é uma medida recomendada pelo Ministério da Saúde, devido a relatos de estudos científicos demonstrando que, mesmo tratados, os animais se mantêm como reservatórios, abrigando o parasito em seus organismos, podendo transmiti-los aos insetos vetores. Estudos complementares são necessários para se avaliar a incidência do aumento da LV e uma possível associação ao tratamento canino.

Na Tabela 3 observa-se o percentual de casos de LV na zona rural, com um total de $10,71 \%$ de notificações.

TABELA 3: Frequência de casos de Leishmaniose Visceral no município de Teófilo, classificados pela zona rural do município.

\begin{tabular}{cccc}
\hline Zona Rural & $\begin{array}{c}\text { Número de casos por ano de } \\
\text { notificação }\end{array}$ & TOTAL \\
\hline Rio Pretinho & 2017 & 2018 & \\
C. Santo Antonio & 01 & $\mathrm{x}$ & 01 \\
C. Santa Barbara & 01 & $\mathrm{x}$ & 01 \\
F. Propérsio Leandro & $\mathrm{x}$ & 01 & 01 \\
Serra de Honório & $\mathrm{x}$ & 01 & 01 \\
Topázio & 02 & $\mathrm{x}$ & 02 \\
Poton & 01 & $\mathrm{x}$ & 01 \\
& 01 & $\mathrm{x}$ & 01 \\
\hline
\end{tabular}


Barra Nova

F. Limeira
01

01
$\mathrm{X}$

$\mathrm{X}$
01

01

\section{Total de casos}

notificados na Zona

Rural

FONTE: Dados da própria pesquisa

A análise da distribuição dos casos notificados de acordo com o sexo (Tabela 4) mostra uma incidência maior no sexo masculino $(57,1 \%)$. O sexo feminino teve uma porcentagem inferior de infecção quando comparado com o sexo masculino em todos os anos analisados, exceto no ano de 2017, onde o percentual feminino de infecção foi de $67 \%$ dos casos notificados. Nos estudos de Rodrigues (2008), o autor expõe sobre a incidência de LV no sexo masculino, devido ao fato de uma maior exposição ao flebótomo infectado, através de atividades expostas, como por exemplo, o trabalho agrícola ou em ambientes externos.

TABELA 4: Frequência de casos de Leishmaniose Visceral no município de Teófilo, classificados pelo sexo dos indivíduos infectados.
ANO DE NOTIFICAÇÃO
SEXO
NÚMERO DE CASOS

\begin{tabular}{lll}
\hline & Masculino & 04 \\
2014 & Feminino & 02 \\
& & \\
\hline \multirow{2}{*}{2015} & Masculino & 05 \\
& Feminino & 01 \\
& & \\
\hline
\end{tabular}


FONTE: Dados da própria pesquisa

A análise da distribuição dos casos também foi realizada de acordo com a faixa etária. Assim, foi possível observar (Tabela 5) que a incidência da LV ocorre em todas as idades, com índices mais expressivos em crianças de 1 a 10 anos de idade $(32,1 \%)$ e em adultos de 40 a 50 anos $(17,8 \%)$. O mesmo padrão foi constatado nos estudos de Andrade et al., 2012, Almeida (2011), Rodrigues (2008), Teodoro et al., 2003). O acometimento com maior prevalência em crianças nessa faixa etária pode estar relacionado com sistema imunológico em fase de desenvolvimento nesse grupo (ALMEIDA, 2011). Nos adultos maiores de 40 anos, estes se encontram em fase produtiva de trabalhos, ficando esse grupo mais exposto ao flebótomo no ambiente peridomiciliar, podendo dessa forma infectar-se.

TABELA 5: Frequência de casos de Leishmaniose Visceral no município de Teófilo, classificados por faixa etária dos indivíduos infectados nos anos de 2014-2018.

\section{FAIXA ETÁRIA}

0 a 10

11 a 20

21 a 30

\section{NUMERO DE CASOS}

\section{8}

07

05 
31 a 40

06

41 a 50

10

51 a 60

61 a 70

71 a 80

81 a 90

91 a 100
04

04

$\mathrm{X}$

02

$\mathrm{X}$

FONTE: Dados da própria pesquisa

\section{CONCLUSÃO}

A análise do comportamento das doenças e sua localização no espaço e no tempo constitui-se em importante ferramenta de estudo epidemiológico, visto que proporciona um melhor conhecimento sobre os problemas de saúde regional, com capacidade de indicar os fatores de risco para a população.

A estratificação de riscos oferece subsídios aos gestores e profissionais da saúde para a adoção de ações e direcionamento de áreas prioritárias a serem trabalhadas.

Os resultados relatados neste estudo sumarizam a sumarizam a situação atual da LV em Teófilo Otoni, indicando um padrão epidemiológico semelhante ao da maioria dos focos da doença no Brasil, com expansão geográfica e aumento dos casos humanos. Apesar da situação endêmica para a LV no município de Teófilo Otoni ser antiga, o presente estudo permitiu evidenciar aspectos que precisam ainda ser estudados, como o papel real dos hospedeiros domésticos no ciclo zoonótico de transmissão do agente etiológico da LV. Observa-se que as medidas preconizadas para conter a incidência doença não estão sendo eficazes, apontando para a necessidade de ações 
complementares, como a realização de trabalhos informativos, de forma a incorporar as medidas de controle e prevenção no dia a dia da população.

Por fim, o presente estudo pode servir como subsídio para os planejamentos de futuras estratégias de prevenção ou controle da LV na região de Teófilo Otoni - MG.

\section{REFERÊNCIAS}

ALMEIDA, A. S. Identificação de áreas sob maior risco para leishmaniose visceral, na cidade de Teresina, Piauí Brasil. Rio de Janeiro, 2011. 111f. Tese (Doutorado em Saúde Coletiva) - Universidade do Estado do Rio de Janeiro, Instituto de Medicina Social, 011.

ANDRADE FILHO, J. D.; GALATI, E. A. B.; FALCÃO, A. L. Nyssomyia intermedia and Nyssomyia neivai (Diptera: Psychodidae: Phlebotominae) geographicaldistribution and epidemiological importance. Memórias do Instituto Oswaldo Cruz, Rio de Janeiro, v. 102, n. 4, p. 481-487, Jun 2007.

ANDRADE, T. A. S. et al. Perfil epidemiológico dos casos notificados de leishmaniose tegumentar americana no município de Igarassu (PE) no período de 2008 a 2010. Scire Salutis, Aquidabã, v. 2, n. 2, pp. 5-15, 2012.

BASANO, S. A.; CAMARGO, L. M. A. Leishmaniose tegumentar americana: histórico, epidemiologia e perspectivas de controle. Revista Brasileira de Epidemiologia, São Paulo, v. 7, n. 3, pp. 328-337, 2004.

BRANDÃO-FILHO, SP, SILVA OA, ALMEIDA EL, VALENÇA HF, ALMEIDA FA. Incidência da leishmaniose visceral sem a presença de Lutzomyia longipalpis, na Zona da Mata de Pernambuco, Brasil. Rev Soc Bras Med Trop. 2002; 35 (Supl 1):333.

CHAPPUIS, F.; SUNDAR, S.; HAILU, A.; GHALIB, H.; RIJAL, S.; PEELING, R.W.; ALVAR, J.; BOELAERT, M., 2007. Visceral leishmaniasis: What are the needs for diagnosis, treatment and control?. Nat. Ver. Microbiol, Vol. 5, nº 11, p. 873-82.

CORRÊA, C.N.M., CORRÊA, W.M.C. Enfermidades Infecciosas dos Mamíferos Domésticos. Rio de Janeiro: Médica e Científica Ltda. p. 717 - 720, 1992.

COSTA, C.H.N. How effective is dog culling in controlling zoonotic visceral leishmaniasis? A critical evaluation of the science, politics and ethics behind this public health policy. Rev. Soc. Bras. Med. Trop. Uberaba. 2011Vol. 44, nº 2, p. 232-242. 
COSTA, C.H.N. Characterization and speculations on the urbanization of visceral leishmaniasis in Brazil. Caderno de Saúde Pública, v.24, n.12, p.2959-2963, 2008. Fev. 2008. doi: 10.1590/S0102-311X2008001200027.

HARTZ, Z.M.A., CONTANDRIOPOULOS, A.P., 2004. Integralidade da atenção e integração de serviços de saúde: desafios para avaliar a implantação de um "sistema sem muros”. Cad. Saúde Pública, Vol. 20, n² 2, p. 331- 336.

IBGE - Instituto Brasileiro de Geografia e Estatística. Teófilo Otoni. 2014. Disponível em:

<www.cidades.ibge.gov.br/painel/historico.php?lang=\&codmun=316860\&search=mina s-gerais|teofilo-otoni|infograficos:-historico>. Acesso em: 22 de maio. 2019.

MARTINS, Marcos Lobato. Ocupação e desflorestamento numa área de fronteira: Vale do Mucuri, MG - 1890 a 1950. Revista de História Regional 15(1): 40-77, Verão, 2010.

MARZOCHI, M. C. A. Leishmanioses no Brasil: as leishmanioses tegumentares. Jornal Brasileiro de Medicina, Rio de Janeiro, v. 63, n. 5/6, pp. 82-104, 1992.

MENDES, E.V., 2010. As redes de atenção à saúde. Cien Saude Colet, Vol. 15, nº 5, p. 2297-305.

NUNES, W. S. et al. Epidemiological profile of leishmaniasis at a reference service in the state of Alagoas, Brazil, from January 2000 to September 2008. Brazilian Journal of Infectious Diseases, Salvador, v. 14, n. 4, p. 342-345, 2010.

ORGANIZAÇÃO MUNDIAL DE SAÚDE. Leishmaniose: Informe epidemiológico das Américas. 2019. Disponível em: < http://portal.cfmv.gov.br/uploads/files/2019cde-leish-informe-epi-das-americas.pdf>. Acesso em: 22 maio. 2019.

ORGANIZAÇÃO PAN-AMERICANA DA SAÚDE-OPAS/OMS: Leishmanioses: Informe Epidemiológico nas Américas: Washington: Organização Pan-Americana da Saúde; 2019 Disponível em: <http://iris.paho.org/xmlui/bitstream/handle/123456789/34857/LeishReport6_por.p df?sequence=5 >. Acesso em maio. 2019. 
RODRIGUES, A. C. E. Características Epidemiológicas e Distribuição Espacial da Enzootia Canina de Leishmaniose Visceral na Cidade de Teresina - Piauí, no período de 2003 - 2006. 101f. 2008. Dissertação (Mestrado em Saúde Pública). Fundação Oswaldo Cruz, Teresina, 2008.

RONDON, F.C.M; Estudo transversal da Leishmaniose Visceral Canina na cidade de Fortaleza, Ceará, Brasil, 2007. Disponível em:

<http://www.uece.br/ppgcv2/dmdocumentes/fernanda rondon.pdf > Acesso em: 25/09/2011.

SHAW, J. J. et al. Leishmaniasis in Brazil XXIII. The identification of Leishmania braziliensis in wild-caught sandflies, using monoclonal antibodies. American Journal of Tropical Medicine Hygiene, Baltimore, v. 81, n. 1, p. 69-72, 1987.

SILVA, C. M. S. et al. Seminário Piauiense: Educação e Contexto. Campina Grande: Triunfal Gráfica e Editora, 2010.

SILVA ES, Gontijo CMF, Pacheco RS, Fiuza VOP, Brazil RP. Visceral Leishmaniasis in the Metropolitan Region of Belo Horizonte, State of Minas Gerais, Brazil. Mem Inst Oswaldo Cruz 2001; 3: 285-91.

SILVA, J. G. D. et al. Infecção natural de Lutzomyia longipalpis por Leishmania sp. em Teresina, Piauí, Brasil. Cadernos de Saúde Pública, Rio de Janeiro, v. 23, n. 7, p. 1715- 1720, jul. 2007.

TEODORO, U. et al. Influência da reorganização, da limpeza do peridomicílio e da desinsetização de edificações na densidade populacional de flebotomíneos, no município de Doutor Camargo, Estado do Paraná, Brasil. Cadernos de Saúde Pública, Rio de Janeiro, v. 19, pp. 1801-1813, 2003.

VERONESI, R., FOCACCIA, R. Tratado de Infectologia, São Paulo: Editora Atheneu, 2V., 1996.

WORLD HEALTH ORGANIZATION. WHO. Special Programme for Research and Training in Tropical Diseases. 2012. (WHO Technical Report Series, No. 975, 2012). Disponível em: 〈http://www.who.int/tdr/index.html > Acesso em: 022 maio. 2012.

WORLD HEALTH ORGANIZATION-WHO. Working to overcome the global impact of neglected tropical diseases: 2011. First WHO report on neglected tropical diseases. Expert Committee on the Control of Leishmaniasis. Geneva: World Health Organization. Disponível em: < 
https://www.who.int/neglected_diseases/2010report/WHO_NTD_report_update_2011.p df. Acesso em: maio. 2019.

YOUNG, D. G.; DUNCAN, M. A. Guide to the identification and geographic distribution of Lutzomyia sand flies in Mexico, the West Indies, Central and South America (Diptera: Psychodidae). Memoirs of the American Entomological Institute, Gainesville, v. 54, p. 1- 881, 1994. 